\title{
Stellungnahme zur Chirurgie im privaten und im öffentlichen Spital, Kodex für die Honorarabrechnung
}

Ehrenrat der Schweizerischen Gesellschaft für Chirurgie*

* Mitglieder des SGC-Ehrenrates: Prof. Dr. Hans Stirnemann, PD Dr. Urs Neff

Prof. Dr. J.-C. Givel.

Korrespondenz:

Schweizerische Gesellschaft für Chirurgie

Netzibodenstrasse 34

Postfach 1527

CH-4133 Pratteln
Die vorliegende Stellungnahme des Ehrenrates der Schweizerischen Gesellschaft für Chirurgie beschreibt die wesentlichen Unterschiede und Aufgabenbereiche der Chirurgie in privaten und öffentlichen Spitälern zum besseren Verständnis zuhanden einer breiteren Öffentlichkeit.

Der Anhang «Kodex für Honorarabrechnung» gibt die wichtigsten Voraussetzungen wieder, die zur Verrechnung von Honorare bei Privat- und Halbprivatpatienten berechtigen.

Diese Stellungnahme wurde am 21. November 2002 vom SGC-Vorstand verabschiedet und gilt als Empfehlung für die Mitglieder der SGC.

Operation, Nachbehandlung und Pflege von Patienten sind für Privat- und öffentliche Spitäler zentrale Aufgabenbereiche. Diese werden nach gleichen Prinzipien wahrgenommen und erfüllt. In fachlicher und in qualitativer Beziehung besteht kein Unterschied. Das Wohl des Kranken und die Bemühungen für seine Gesundheit sind oberstes Gebot. Dies ist nicht zuletzt Folge eines gesunden Konkurrenzverhältnisses zwischen Spitälern. Die Praxis der «second opinion» wird für beide Spitaltypen unterstützt, sofern sie der Beseitigung von Unsicherheiten und der besseren Information des Patienten dienen.

Unterschiedliche Strukturen und Organisationsformen sowie verschiedene Kostenträger führen jedoch bei den zwei Spitaltypen in diversen Bereichen zu nennenswerten Unterschieden.

\section{Arztwahl}

Im Privatspital besteht, für entsprechend versicherte Patienten, grundsätzlich die freie Arztwahl.

Im öffentlichen Spital gilt die freie Arztwahl uneingeschränkt nur für Halbprivatpatienten und Privatpatienten.

\section{Behandlung}

Im Privatspital ist der vom Patienten gewählte Arzt für die Behandlung zuständig und verantwortlich (sogenanntes Belegarztsystem).

Im öffentlichen Spital ist es, je nach Komplexität des Problems und des Versicherungsstatus der Patienten, der Chefarzt, ein Oberarzt oder, bei einfachen Situationen, ein Assistenzarzt (in der Regel unter Anleitung eines erfahrenen Kollegen), der die Behandlung durchführt. Immer trägt aber letztlich der Chefarzt die Gesamtverantwortung. Man spricht hier von einem Delegationsprinzip.

\section{Fachbereiche}

Privatspitäler können ihre Tätigkeit auf bestimmte Fachbereiche beschränken.

Die öffentlichen Spitäler müssen alle von den Behörden übertragenen Fachbereiche mit deren Aufgaben übernehmen und erfüllen, was eine vollständige Versorgung im Spitalbereich gewährleistet.

\section{Aufnahmepflicht}

Das Privatspital ist nicht verpflichtet, alle Patienten aufzunehmen, d.h. es kann seine Patienten nach medizinischen und versicherungstechnischen Kriterien auswählen.

Beim öffentlichen Spital besteht jedoch eine generelle Aufnahmepflicht aller Patienten (inklusive Notfallpatienten), ungeachtet ihres medizinischen Problems oder ihrer Versicherungsklasse. Dies bringt mit sich, dass öffentliche Spitäler auch für sozial oder medizinisch schwierige Fälle zuständig sind, was sie finanziell belastet. 


\section{Lehre, Forschung und}

\section{Qualitätsmanagement}

Öffentliche Spitäler haben von Staates wegen die Verpflichtung zur Ausbildung von Studenten und Ärzten und sorgen damit für den unerlässlichen beruflichen Nachwuchs. Dies trifft für Privatspitäler nur in Einzelfällen zu.

In grösseren öffentlichen Spitälern und Universitätskliniken besteht zusätzlich die Verpflichtung zu klinischer Forschungstätigkeit, was für den Fortschritt des Fachgebietes unerlässlich ist.

Für öffentliche Spitäler besteht zudem eine Verpflichtung für Qualitätsmanagement.

\section{Finanzierung}

Die im allgemeinen Interesse erbrachten Leistungen eines öffentlichen Spitals werden durch staatliche Subventionen mitfinanziert. Im Privatspital erfolgt dagegen in der Regel keine finanzielle Unterstützung durch die öffentliche Hand.

\section{Anhang 1}

\section{Kodex für Honorarabrechnung} bei Halbprivat- und Privatpatienten

- Der Eingriff wird vom Operateur mit dem Patienten besprochen.

- Der Eingriff wird vom Operateur persönlich durchgeführt.

- Dies bedeutet, dass der Operateur alle wesentlichen und kritischen Schritte selbst durchführt oder in lehrender Funktion persönlich anwesend assistiert. $\mathrm{Zu}$ diesen gehören alle vor, während und nach dem Eingriff zu treffenden Indikationen und Entscheide mit unmittelbaren Auswirkungen auf das Vorgehen und die von der Erfahrung und der Fähigkeit des Operateurs abhängigen technischen Schritte («ability» und «skills»).

- Wird der Operateur während der Operation zu einem Notfall oder einem Problem in einen anderen Operationssaal gerufen, kann die Weiterführung einem Oberarzt oder einem erfahrenen Assistenten für die Dauer der Abwesenheit delegiert werden. Der Operateur bleibt aber auch während solcher Ausnahmesituationen verantwortlich.

- Das Ergebnis des Eingriffes wird dem Patienten vom Operateur persönlich mitgeteilt. 